\title{
ON THE GLOBAL STABILITY OF A DELAY EPIDEMIC MODEL
}

\author{
XIAODONG LIN ${ }^{1}$ \\ (Received 19 September 1990; revised 18 February 1991)

\begin{abstract}
In this paper, we study the asymptotic behavior of an SIRS epidemic model with a time delay in the recovered class and a nonlinear incidence rate. A conjecture of Hethcote et al. [5] on the global stability of the disease-free equilibrium is solved. Moreover, we analyse the model when the contact number takes its threshold value. We show that solutions tend to either the disease-free equilibrium or to a unique positive endemic equilibrium, and there is no periodic solution.
\end{abstract}

\section{Introduction}

To study the spread of an infectious disease in a constant population, one usually divides the total population into three disjoint classes: susceptible, infectious and recovered (removed). In the susceptible class, individuals can incur the disease but are not yet infected. The infectious class consists of those who are transmitting the disease to others. The recovered class consists of individuals who have recovered from the disease and have temporary immunity. We denote the fraction of the population in each class at time $t$ by $S(t), I(t)$ and $R(t)$, respectively.

In recent years, various epidemic models (so called SIRS models) have been formulated to investigate such interactions between these three classes. Hethcote [2] studied a model with a bilinear incidence rate $\beta I S$, where $\beta$ is the contact rate. Hethcote et al. [3] studied a model with a bilinear incidence rate and a time delay in the recovered class, which means individuals gain a period of temporary immunity right after recovering. Liu et al. [6] considered a model with a nonlinear incidence rate of form $\beta I^{p} S^{q}$, where

\footnotetext{
${ }^{1}$ Dept. of Applied Maths, University of Waterloo, Waterloo, Ontario, Canada N2L 3G1.

(C) Copyright Australian Mathematical Society 1992, Serial-fee code 0334-2700/92
} 
$p$ and $q$ are positive numbers, but without time delay. The model we will study here was proposed by Hethcote $e t$ al. [5]. A constant time delay and a nonlinear incidence rate are taken into account in this model. It is assumed that the incidence rate is nonlinear and is of the form $\beta I^{p} S$. The recovery rate of individuals in the infectious class is proportional to the infectious fraction at rate $\gamma$, and then the average infectious period is $1 / \gamma$. Furthermore, a constant period, $\omega$, of temporary immunity is introduced, so that the probability $P(t)$ of remaining immune $t$ time units after recovering is 1 for $0 \leq t \leq \omega$, and 0 for $t>\omega$. With the above assumptions, they obtained the following delay epidemic model

$$
\begin{gathered}
I^{\prime}(t)=-\gamma I(t)+\beta I^{p}(t) S(t), \\
R(t)=R_{0}(t)+\gamma \int_{0}^{t} I(x) P(t-x) d x, \\
S^{\prime}(t)= \begin{cases}-R_{0}^{\prime}(t)-\beta I^{p}(t) S(t), & \text { for } t \leq \omega, \\
\gamma I(t-\omega)-\beta I^{p}(t) S(t), & \text { for } t>\omega,\end{cases}
\end{gathered}
$$

where $R_{0}(t)$ is the fraction of the initial population which is initially in the recovered class, and is still in at time $t$. It is reasonable to assume $R_{0}(t)$ is a differentiable, nonincreasing function with $R_{0}(t)=0$ for $t>\omega$.

In [5], Hethcote et al. analysed the model (1.1)-(1.3) to determine the equilibria and examine their stability by varying the index $p$ and the contact number $\sigma=\beta / \gamma$. For the case $p>1$ in particular, they proved the following results.

THEOREM 2.2. Let $\sigma^{*}=p^{p}(1+r)^{p-1} /(p-1)^{p-1}$ where $r=\gamma \omega$. For (1.1)(1.3), if

(i) $p>1$, then the disease-free equilibrium is locally asymptotically stable. In addition, if $\sigma<\sigma^{*}$, it has no positive equilibrium;

(ii) $p>1$ and $\sigma=\sigma^{*}$, then it has one positive equilibrium;

(ii) $p>1$ and $\sigma>\sigma^{*}$, then it has two positive equilibria and the smaller positive equilibrium is unstable. The stability of the larger positive equilibrium depends on the value of $p, \sigma, r$, and there is a Hopf bifurcation near it for some parameter values.

Moreover, they gave a sufficient condition for the global stability of the disease-free equilibrium. They showed that there is a positive $\sigma_{1}=$ $p^{p} /(p-1)^{p-1}$ such that if $\sigma<\sigma_{1}$, the disease-free equilibrium is globally asymptotically stable. They further conjectured that the disease-free equilibrium is globally asymptotically stable for all $\sigma<\sigma^{*}$.

In the present paper, we solve this conjecture. Also, we analyse the asymptotic behavior of solutions of the model (1.1)-(1.3) as the contact number $\sigma$ 
takes the threshold value $\sigma^{*}$. The results we prove show that there is no periodic solution when $\sigma=\sigma^{*}$. Every solution tends to either the disease-free equilibrium or to a unique positive endemic equilibrium.

The organisation of this paper is as follows. In this section, we show the reduction in [5] of the model (1.1)-(1.3) to an equivalent scalar integrodifferential equation. In Section 3, we prove the conjecture. In Section 4, we use the techniques developed in Section 3 to analyse the asymptotic behavior of the model when $\sigma=\sigma^{*}$. In the final section, we discuss our results and compare them with the results of Hethcote $e t$ al.

The model (1.1)-(1.3) can be reduced to an equivalent scalar integrodifferential equation, namely for $t \leq \omega$,

$$
I^{\prime}(t)=-\gamma I(t)+\beta I^{p}(t)\left[1-I(t)-R_{0}(t)-\gamma \int_{0}^{t} I(x) d x\right],
$$

and for $t>\omega$,

$$
I^{\prime}(t)=-\gamma I(t)+\beta I^{p}(t)\left[1-I(t)-\gamma \int_{t-\omega}^{t} I(x) d x\right] .
$$

Obviously, $I(t) \equiv 0$ is always an equilibrium of $(1.4)-(1.5) . \quad((S, I, R)=$ $(1,0,0)$ corresponding to $(1.1)-(1.3)$.) This is called the disease-free equilibrium. Studying the stability of equilibria of the model (1.1)-(1.3) can be done by studying the stability of equilibria of (1.4)-(1.5). The initial-value problem of $(1.4)-(1.5)$ is well-posed, and the interval $[0,1]$ is a positive invariant set and an attractive region for all nonnegative solutions. Moreover, the maximal interval for every nonnegative solution is $[0, \infty)$ (cf. [5]).

2. Global stability of the disease-free equilibrium when $p>1$ and $\sigma<\sigma^{*}$

In this section, we study the asymptotic behavior of solutions of (1.4)(1.5) when $p>1$ and $\sigma<\sigma^{*}$. We prove that if $p>1$ and $\sigma<\sigma^{*}$, all nonnegative solutions will approach to the disease-free equilibrium. As a consequence, the conjecture of Hethcote et al. is verified. First, we introduce a definition, which will be used in our proofs.

Definition. Let $f(t), t \in[0, \infty)$, be a continuous function. $f(t)$ is said to be eventually nonincreasing if there is a positive $T>0$ such that $f(t)$ is nonincreasing for $t \geq T$.

We now state and prove our results. 
THEOREM 2.1. Suppose $p>1$ and $\sigma<\sigma^{*}$. Then, every nonnegative solution of (1.4)-(1.5) is an eventually nonincreasing function and it tends to the disease-free equilibrium.

To prove Theorem 2.1, we need the following lemmas.

Let $I(t)$ be a nonnegative solution of $(1.4)-(1.5)$. Since $[0,1]$ is the attractive region, we assume $0 \leq I(t) \leq 1$ without loss of generality. Define

$$
\delta_{n}=\min \{I(t) ; t \in[(n-1) \omega, n \omega]\}, \quad n=1,2, \ldots
$$

Then, for each $n$, there is a $t_{n} \in[(n-1) \omega, n \omega]$ such that $I\left(t_{n}\right)=\delta_{n}$.

Lemma 2.1.1. Suppose $p>1$ and $\sigma \leq \sigma^{*}$. Then, $I(t)$ is an eventually nonincreasing function or $t_{n} \neq n \omega$ for $n=1,2, \ldots$.

Proof. If $t_{n}=n \omega$ for some $n$, then $I\left(t_{n}\right) \leq I(t), t \in[(n-1) \omega, n \omega]$. Thus

$$
\begin{aligned}
I^{\prime}\left(t_{n}\right) & =-\gamma I\left(t_{n}\right)+\beta I^{p}\left(t_{n}\right)\left[1-I\left(t_{n}\right)-\gamma \int_{t_{n}-\omega}^{t_{n}} I(x) d x\right] \\
& \leq-\gamma I\left(t_{n}\right)+\beta I^{p}\left(t_{n}\right)\left[1-(1+r) I\left(t_{n}\right)\right] \\
& \leq \gamma I\left(t_{n}\right)\left(-1+\frac{\sigma}{\sigma^{*}}\right) .
\end{aligned}
$$

The last two inequalities become equalities if and only if $I(t) \equiv 0$ or $I(t) \equiv$ $(p-1) / p(1+r)$. In these cases, $I(t)$ is already an eventually nonincreasing function. Hence, we assume that $I(t)$ is not either one. So, we have $I^{\prime}\left(t_{n}\right)<0$. This implies that there is a $t^{\prime}>t_{n}$ such that $I(t)$ is a strictly decreasing function on $\left[t_{n}, t^{\prime}\right)$.

Let

$$
T_{n}=\sup \left\{t^{\prime} ; I(t) \text { strictly decreases on }\left[t_{n}, t^{\prime}\right)\right\} \text {. }
$$

If $T_{n}<\infty$, then $T_{n}$ is a minimal point of $I(t)$. Thus, $I^{\prime}\left(T_{n}\right)=0$, and $I\left(T_{n}\right)<I(t)$ on $\left[t_{n}-\omega, T_{n}\right)$. In particular, $I\left(T_{n}\right) \leq I(t)$ and $I\left(T_{n}\right) \neq I(t)$, for $t \in\left[T_{n}-\omega, T_{n}\right]$. Therefore, we have

$$
\begin{aligned}
I^{\prime}\left(T_{n}\right) & =-\gamma I\left(T_{n}\right)+\beta I^{p}\left(T_{n}\right)\left[1-I\left(T_{n}\right)-\gamma \int_{T_{n}-\omega}^{T_{n}} I(x) d x\right] \\
& <-\gamma I\left(T_{n}\right)+\beta I^{p}\left(T_{n}\right)\left[1-(1+r) I\left(T_{n}\right)\right] \\
& \leq \gamma I\left(T_{n}\right)\left(-1+\frac{\sigma}{\sigma^{*}}\right) \leq 0,
\end{aligned}
$$

which contradicts $I^{\prime}\left(T_{n}\right)=0$.

Hence, $T_{n}=\infty$. Consequently, $I(t)$ is an eventually nonincreasing function. We complete the proof. 
Lemma 2.1.2. Suppose $p>1$ and $\sigma \leq \sigma^{*}$. If $t_{n} \neq n \omega$, for $n=1,2, \ldots$, then $\delta_{n+1}>\delta_{n}$ for $n=1,2, \ldots$.

Proof. If $\delta_{n+1} \leq \delta_{n}$, then $\delta_{n+1}$ is the minimal value of $I(t)$ in $[(n-1) \omega$, $(n+1) \omega]$. From $t_{n+1} \neq(n+1) \omega, t_{n} \in((n-1) \omega,(n+1) \omega)$. Thus $I^{\prime}\left(t_{n+1}\right)=0$. Again, we have

$$
\begin{aligned}
I^{\prime}\left(t_{n+1}\right) & =-\gamma I\left(t_{n+1}\right)+\beta I^{p}\left(t_{n+1}\right)\left[1-I\left(t_{n+1}\right)-\gamma \int_{t_{n+1}-\omega}^{t_{n+1}} I(x) d x\right] \\
& <\gamma I\left(t_{n+1}\right)\left(-1+\frac{\sigma}{\sigma^{*}}\right) \leq 0,
\end{aligned}
$$

a contradiction to $I^{\prime}\left(t_{n+1}\right)=0$. Hence this lemma is proved.

LEMMA 2.1.3. Under the condition of Lemma 2.1.2, we have

$$
\delta_{n+1}-\delta_{n}>\frac{1}{\sigma r}\left(1-\frac{\sigma}{\sigma^{*}}\right) \text {. }
$$

Proof. Since $t_{n+1}$ is a minimal point in $[n \omega,(n+1) \omega]$ and $t_{n+1} \neq(n+1) \omega$, $I^{\prime}\left(t_{n+1}\right) \geq 0$. On the other hand,

$$
I^{\prime}\left(t_{n+1}\right)=-\gamma I\left(t_{n+1}\right)+\beta I^{p}\left(t_{n+1}\right)\left[1-I\left(t_{n+1}\right)-\gamma \int_{t_{n+1}-\omega}^{t_{n+1}} I(x) d x\right] .
$$

By Lemma 2.1.2, $\delta_{n}$ is the minimal value in $[(n-1) \omega,(n+1) \omega]$. Hence,

$$
\begin{aligned}
I^{\prime}\left(t_{n+1}\right) & <-\gamma \delta_{n+1}+\beta \delta_{n+1}^{p}\left[1-\delta_{n+1}-r \delta_{n}\right] \\
& =\gamma \delta_{n+1}\left\{-1+\sigma \delta_{n+1}^{p-1}\left[1-(1+r) \delta_{n+1}+r\left(\delta_{n+1}-\delta_{n}\right)\right]\right\} .
\end{aligned}
$$

From $I^{\prime}\left(t_{n+1}\right) \geq 0$,

$$
\begin{aligned}
\sigma r\left(\delta_{n+1}-\delta_{n}\right) & \geq \sigma r \delta_{n+1}^{p-1}\left(\delta_{n+1}-\delta_{n}\right) \\
& >1-\sigma \delta_{n+1}^{p-1}\left[1-(1+r) \delta_{n+1}\right] \\
& \geq 1-\frac{\sigma}{\sigma^{*}}
\end{aligned}
$$

i.e.

$$
\delta_{n+1}-\delta_{n}>\frac{1}{\sigma r}\left(1-\frac{\sigma}{\sigma^{*}}\right) .
$$

LEMMA 2.1.4. If $p>1$ and $\sigma<\sigma^{*}$, then $I(t)$ is an eventually nonincreasing function.

Proof. If not, by Lemmas 2.1.1 and 2.1.3,

$$
\delta_{n+1}-\delta_{n}>\frac{1}{\sigma r}\left(1-\frac{\sigma}{\sigma^{*}}\right)>0 .
$$


Thus, $\lim _{n \rightarrow \infty} \delta_{n}=\infty$, which is impossible because $I(t)$ is bounded. Therefore we prove the lemma.

Proof of Theorem 2.1. From Lemma 2.1.4, we know that every nonnegative solution $I(t)$ of (1.4)-(1.5) is eventually nonincreasing. Let $I_{0}$ denote the limit of $I(t)$, as $t \rightarrow \infty$. Since $I(t)$ and $I^{\prime}(t)$ are bounded, we have $\lim _{t \rightarrow \infty} I^{\prime}(t)=0$. Hence, let $t \rightarrow \infty$ in (1.5); we obtain

$$
-\gamma I_{0}+\beta I_{0}^{p}\left[1-(1+r) I_{0}\right]=0 .
$$

But, for $p>1$ and $\sigma<\sigma^{*}$, only $I \equiv 0$ satisfies this equality. Hence $I_{0}=0$. This implies

$$
\lim _{t \rightarrow \infty} I(t)=0 .
$$

Therefore, the disease-free equilibrium is globally asymptotically stable to all nonnegative solutions for (1.4)-(1.5).

\section{Asymptotic behavior of (1.4)-(1.5) when $p>1$ and $\sigma=\sigma^{*}$}

It is easy to show that in the case $p>1$ and $\sigma=\sigma^{*}$, the unique positive equilibrium is degenerate, i.e. the characteristic equation for its linear variational equation has a zero eigenvalue. Hence, the stability of the positive equilibrium becomes very difficult to determine. In this section, we will show that if a nonnegative solution does not approach the positive equilibrium, it must approach the disease-free equilibrium. In other words, the feasible region is split into two disjoint regions: the attractive region of the positive equilibrium and the attractive region of the disease-free equilibrium. Furthermore, we give an estimation of the attractive region of the disease-free equilibrium.

When $p>1$ and $\sigma=\sigma^{*},(1.5)$ has two equilibria: the disease-free equilibrium $I=0$ and the positive equilibrium $I=I_{e}=(p-1) / p(1+r)$. In this situation, we have the following.

THEOREM 3.1. Let $I(t)$ be a nonnegative solution of (1.4)-(1.5). Then, there are only two possibilities:

(i) $I(t)$ is an eventually decreasing function and tends to one of the equilibria,

(ii) $I(t)$ is a damped oscillation and $\lim _{t \rightarrow \infty} I(t)=I_{e}$.

Therefore, there is no periodic solution for (1.4)-(1.5).

Proof. If $I(t)$ is an eventually nonincreasing function, proceeding as in the proof of Theorem 2.1, I(t) tends to one of the equilibria. Hence, (i) is true. 
If $I(t)$ is not an eventually nonincreasing function, by Lemma 2.1.2, $\delta_{n+1}>\delta_{n}$ for $n=1,2 \ldots$.

Thus, there is no periodic solution in either case.

We now prove that if $I(t)$ is not eventually nonincreasing, $\lim _{t \rightarrow \infty} I(t)$ $=I_{e}$.

First, we claim that $\liminf _{t \rightarrow \infty} I(t)=I_{e}$.

Otherwise, since $\left\{\delta_{n}\right\}_{n=1}^{\infty}$ is a strictly increasing sequence, by Lemma 2.1.2, there is a positive $\mu$ such that $\left|\delta_{n}-I_{e}\right| \geq \mu, n \geq N$ for some integer $N$. Noting that the function $y^{p-1}-(1+r) y^{p}$ takes the unique maximal value $1 / \sigma^{*}$ at $y=I_{e}$, we have

$$
\delta_{n+1}^{p-1}-(1+r) \delta_{n+1}^{p} \leq \frac{1-\varepsilon}{\sigma^{*}}, \quad n \geq N,
$$

for some positive $\varepsilon$.

Thus, from the proof of Lemma 2.1.3,

$$
\begin{aligned}
\delta_{n+1}-\delta_{n} & >\frac{1}{\sigma^{*} r}\left\{1-\sigma^{*} \delta_{n+1}^{p-1}\left[1-(1+r) \delta_{n+1}\right]\right\} \\
& \geq \frac{\varepsilon}{\sigma^{*} r}>0 .
\end{aligned}
$$

Hence, $I(t)$ is unbounded, which is impossible. Therefore, we must have

$$
\liminf _{t \rightarrow \infty} I(t)=I_{e} \text {. }
$$

By defining that

$$
\varepsilon_{n}=\max \left\{I(t) ; t \in\left[t_{n}-\omega, t_{n}\right]\right\}, \quad n=1,2, \ldots,
$$

there is $s_{n} \in\left[t_{n}-\omega, t_{n}\right]$ such that $I\left(s_{n}\right)=\varepsilon_{n}$. We now claim $\lim _{n \rightarrow \infty} \varepsilon_{n}$ $=I_{e}$.

If not, then there is a $\mu>0$ and a subsequence, assumed to be $\left\{s_{n}\right\}_{n=1}^{\infty}$ itself, without loss of generality, such that $I\left(s_{n}\right)>I_{e}+3 \mu$. Observing from (1.5) that $I^{\prime}(t)$ is bounded, $I(t)$ is a Lipschitz function. Hence, there is a $\alpha>0$, independent of $n$, such that $\left[t_{n}-\omega, t_{n}\right]$ contains a subinterval $\Delta_{n}$ whose length is greater than $\alpha$ and on which $I(t)>I_{e}+2 \mu$. Since $\lim _{n \rightarrow \infty} \delta_{n}=I_{e}$, there is an integer $N$ such that $I(t)>\delta_{n}+\mu$ for $t \in \Delta_{n+1}$, and $n>N$. Thus we have

$$
\begin{aligned}
I^{\prime}\left(t_{n+1}\right) & =\gamma I\left(t_{n+1}\right)\left\{-1+\sigma^{*} I^{p-1}\left(t_{n+1}\right)\left[1-I\left(t_{n+1}\right)-\gamma \int_{t_{n+1}-\omega}^{t_{n+1}} I(x) d x\right]\right\} \\
& <\gamma \delta_{n+1}\left[-1+\sigma^{*} \delta_{n+1}^{p-1}\left(1-\delta_{n+1}-r \delta_{n}-\gamma \alpha \mu\right)\right] .
\end{aligned}
$$

Because

$$
\lim _{n \rightarrow \infty}\left[-1+\sigma^{*} \delta_{n+1}^{p-1}\left(1-\delta_{n+1}-r \delta_{n}-\gamma \alpha \mu\right)\right]=-\sigma^{*} \gamma \alpha \mu I_{e}^{p-1}<0,
$$

for sufficiently large $n, I^{\prime}\left(t_{n}\right)<0$, which contradicts that $t_{n+1} \neq(n+1) \omega$. 
Therefore, $\lim _{n \rightarrow \infty} \varepsilon_{n}=I_{e}$. From $t_{n+1} \neq(n+1) \omega, n \omega \in\left[t_{n+1}-\omega, t_{n+1}\right]$. We in particular have $\lim _{n \rightarrow \infty} I(n \omega)=I_{e}$.

Third, we claim that for any $t_{0} \geq 0, \lim _{n \rightarrow \infty} I\left(t_{0}+n \omega\right)=I_{e}$.

Obviously, the function $\hat{I}(t)=I\left(t+t_{0}\right)$ is a solution of (1.5). Hence, the lemmas in the last section and the above statements remain true for $\hat{I}(t)$, especially, $\lim _{n \rightarrow \infty} \hat{I}(n \omega)=I_{e}$. This implies $\lim _{n \rightarrow \infty} I\left(t_{0}+n \omega\right)=I_{e}$.

Finally, we prove that $\lim \sup _{t \rightarrow \infty} I(t)=I_{e}$.

If there is $\mu>0$ such that $\limsup _{t \rightarrow \infty} I(t)>I_{e}+\mu$, then there is a sequence $\left\{s_{n}\right\}_{n=1}^{\infty}$ such that $s_{n} \rightarrow \infty$, as $n \rightarrow \infty$, and $I\left(s_{n}\right)>I_{e}+\mu$. Suppose $s_{n}=k_{n} \omega+s_{n}^{\prime}$, where $k_{n} \geq 0$ is an integer and $s_{n}^{\prime} \in[0, \omega)$. Then there is $t_{0} \in[0, \omega]$ and a subsequence of $\left\{s_{n}^{\prime}\right\}$, denoted by $\left\{s_{n}^{\prime}\right\}$ again w.l.o.g., such that $\lim _{n \rightarrow \infty} s_{n}^{\prime}=t_{0}$. Hence, $\lim _{n \rightarrow \infty}\left|t_{0}+k_{n} \omega-s_{n}\right|=0$. Recalling that $I(t)$ is a Lipschitz function,

$$
\lim _{n \rightarrow \infty} I\left(t_{0}+k_{n} \omega\right)=\lim _{n \rightarrow \infty} I\left(s_{n}\right) \geq I_{e}+\mu>I_{e}
$$

This is impossible because $\lim _{n \rightarrow \infty} I\left(t_{0}+n \omega\right)=I_{e}$. Therefore,

$$
\limsup _{t \rightarrow \infty} I(t)=I_{e} \text {. }
$$

With $\liminf _{t \rightarrow \infty} I(t)=I_{e}$, we have $\lim _{t \rightarrow \infty} I(t)=I_{e}$. Our proof is completed.

It is now natural to ask how to locate the attractive regions of both equilibria. In general, it is difficult to do so, partially because they depend on $R_{0}(t)$, the fraction of the initial population which is initially recovered and still stays in the recovered class at time $t$. But the next theorem gives us some information about the attractive region of the disease-free equilibrium.

It is easy to see the equation $I^{p-1}-I^{p}=1 / \sigma^{*}$ has exactly two positive roots. Let $I_{1}$ be the smaller root. Then we have

THEOREM 3.2. Let $I(t)$ be a nonnegative solution of (1.4)-(1.5). If there is $t_{0}$ such that $I\left(t_{0}\right) \in\left[0, I_{1}\right]$, then $I(t) \in\left[0, I_{1}\right]$ for $t \geq t_{0}$ and $\lim _{t \rightarrow \infty} I(t)=$ 0 . In particular, if $I(0) \in\left[0, I_{1}\right], \lim _{t \rightarrow \infty} I(t)=0$. Therefore, $\left[0, I_{1}\right]$ is a positive invariant, attractive region for the disease-free equilibrium.

Proof. Since $I_{1}<I_{e}$, the disease-free equilibrium is the only equilibrium lying in $\left[0, I_{1}\right]$. Hence, it suffices to prove that if $I\left(t_{0}\right) \in\left[0, I_{1}\right]$, then $I(t)$ is a decreasing function for $t \geq t_{0}$. This is true because for any $I(t) \in\left[0, I_{1}\right]$,

$$
\begin{aligned}
I^{\prime}(t) & =-\gamma I(t)+\beta I^{p}(t)\left[1-I(t)-\gamma \int_{t-\omega}^{t} I(x) d x\right] \\
& \leq \gamma I(t)\left[-1+\sigma^{*} I^{p-1}(t)(1-I(t))\right] \leq 0 .
\end{aligned}
$$

The theorem is proved. 


\section{Remarks}

Our results in this paper and Hethcote et al. [3] have shown that for $p \geq 1$ and $\sigma \leq \sigma^{*}$, every feasible solution of the model (1.1)-(1.3) approaches an equilibrium. Hence, there is no periodic solution. Biologically, these results indicate that the disease will die out or the number of individuals in each class will approach a nonzero constant level. In both cases, no periodic outbreaks occur. However, in the other cases, the asymptotic behavior of the model (1.1)-(1.3) is not so simple. By properly choosing parameters, Hethcote et al. [3], [5], showed that the model may possess a Hopf bifurcation. A family of bifurcating periodic solutions therefore arises in these cases.

\section{Acknowledgements}

I would like to thank Professor P. Antonelli for his encouragement.

\section{References}

[1] J. K. Hale, Theory of functional differential equations, (Springer, Berlin, 1977).

[2] H. W. Hethcote, "Qualitative analyses of communicable disease models," Math. Biosci. 28 (1976) 335-356.

[3] H. W. Hethcote, H. W. Stech and P. van den Driessche, "Nonlinear oscillations in epidemic models", SIAM J. Appl. Math. 40 (1981) 1-9.

[4] H. W. Hethcote, H. W. Stech and P. van den Driessche, "Periodicity and stability in epidemic models: A survey," in Differential equations and applications in ecology, epidemics and population problems, (eds. S. W. Busenberg and K. L. Cooke), (Academic Press, New York, 1981) 65-82.

[5] H. W. Hethcote, M. A. Lewis and P. van den Driessche, "An epidemiological model with a delay and a nonlinear incidence rate," J. Math. Biol. 27 (1989) 49-64.

[6] W. M. Liu, H. W. Hethcote and S. A. Levin, "Dynamical behavior of epidemiological models with nonlinear incidence rates," J. Math. Biol. 25 (1987) 359-380.

[7] W. M. Liu, S. A. Levin and Y. Iwasa, "Influence of nonlinear incidence rates upon the behavior of SIRS epidemiological models," J. Math. Biol. 23 (1986) 187-204. 